\title{
Expression of Endothelial-Leukocyte Adhesion Molecule-1 in Elicited Late Phase Allergic Reactions
}

Donald Y. M. Leung, * Jordan S. Pober, ${ }^{*}$ and Ramzi S. Cotran ${ }^{*}$

*Division of Pediatric Allergy-Immunology, The National Jewish Center for Immunology and Respiratory Medicine, Denver, Colorado 80206; and ${ }^{\ddagger}$ Departments of Pathology, Harvard Medical School and Brigham and Women’s Hospital, Boston, Massachusetts 02115

\begin{abstract}
To better understand the events involved in the local migration of inflammatory cells into sites of allergic reactions, we studied expression of the cytokine inducible endothelial cell (EC) neutrophil adhesion molecule, endothelial-leukocyte adhesion molecule (ELAM-1), in sequential skin biopsies from patients with respiratory allergy during the late phase reaction (LPR) between 20 min and until $24 \mathrm{~h}$ after intradermal allergen (ragweed or dust mites) injection. In 7 of 7 atopic patients but in only 1 of 4 apparently normal controls, allergen induced appearance of ELAM-1 on EC. ELAM-1 expression occurred concurrently with the development of inflammatory cell infiltrates by $3-4 \mathrm{~h}$ after intradermal injection. Saline injected sites in all subjects were negative. Skin organ cultures demonstrated that allergen could produce the same EC changes in vitro whether allergen was injected in vivo 20 min before culture or added during skin culture. These EC changes in organ culture were inhibited by the presence of combined anti-sera to both TNF- $\alpha$ and IL-1, but not by antisera to either cytokine alone. We conclude that EC activation occurs in elicited LPR and suggest that cytokineinduced EC activation may play a role in the migration of inflammatory cells into allergic skin reactions. Furthermore, resident cells in the skin rather than infiltrating leukocytes appear to be the source of the cytokines that mediate endothelial activation. (J. Clin. Invest. 1991.87:1805-1809.) Key words: endothelium - adhesion • allergic • skin neutrophil • cytokines
\end{abstract}

\section{Introduction}

Clinically significant allergen-induced reactions are generally characterized by an IgE-dependent biphasic response $(1,2)$. Within minutes of exposure to allergen, mast cells bearing IgE directed to the relevant allergen become activated and release histamine as well as other mediators into local tissue. This immediate reaction is classically evident within 15-60 min of allergen challenge and subsides within 30-90 min later. 3-4 h after allergen challenge, after the immediate reaction has subsided, there is onset of a late phase reaction (LPR) ${ }^{1}$ character-

Portions of this work were presented at the 1990 Annual Meeting of the American Federation of Clinical Research, and appeared in abstract form in 1990. Clin. Res. 38:448.

Received for publication 27 July 1990 and in revised form 24 October 1990.

1. Abbreviations used in this paper: EC, endothelial cell(s); ELAM-1, endothelial-leukocyte adhesion molecule-1; LPR, late phase reaction.

J. Clin. Invest.

(c) The American Society for Clinical Investigation, Inc. $0021-9738 / 91 / 05 / 1805 / 05 \$ 2.00$

Volume 87, May 1991, 1805-1809 ized initially by the infiltration of eosinophils, neutrophils, and mononuclear cells into the inflamed area. Granulocytes reach their maximum cell accumulation at $6-8 \mathrm{~h}$, and by $24-48 \mathrm{~h}$ after onset of the reaction the cellular infiltrate consists predominantly of mononuclear cells.

It has become increasingly appreciated that the IgE-mediated LPR plays an important role in the pathogenesis of chronic allergic diseases. For example, several studies suggest that the chronic cellular infiltrate in asthma may be due to a sustained LPR $(3,4)$. The mechanism for accumulation of inflammatory cells at the site of allergen-induced LPR is unknown. Although mediators such as histamine or leukotriene $\mathrm{C}_{4}$ can induce intercellular leakage of serum macromolecules across endothelial cells (EC) they do not result in the appreciable migration of inflammatory cells into the perivascular compartment. The accumulation of inflammatory cells in injured or antigen-stimulated tissue requires at least two additional events: first, increased adhesion of circulating leukocytes to the luminal surface of venular EC; and second, stimulation of transit of leukocytes across the vascular wall (reviewed in references 5 and 6). The adhesion of leukocytes to endothelial surfaces is mediated by complementary adhesion molecules on the leukocyte and the EC. Cytokines, such as IL-1, TNF, or IFN- $\gamma$, can induce synthesis and expression of specific adhesion molecules on EC that promote attachment of various types of leukocytes $(5,6)$. For example, one such glycoprotein, endothelial-leukocyte adhesion molecule-1 (ELAM-1) is inducible by either IL-1 or TNF, and is involved in the adhesion of PMN to the EC surface $(7,8)$. In this study, we examined whether induction of ELAM-1 is involved in the pathogenesis of cutaneous allergeninduced LPR. The results indicate that EC in skin biopsies from elicited LPR express the cytokine-inducible activation antigen, ELAM-1. Furthermore, ELAM-1 expression may be induced in skin organ culture by addition of allergen in a process dependent on both IL-1 and TNF.

\section{Methods}

Skin biopsies. Skin biopsies were obtained from 2 groups of subjects: first, 15 patients with respiratory allergy (asthma or allergic rhinitis) but no skin disease, all of whom showed immediate and LPR after intradermal injections with either 30 allergenic units of ragweed antigen $E$ or 3 allergenic units of dermatophagoides farinae (dust mite) allergen (Greer Labs, Lenoir, NC); and second, 4 healthy controls with no cutaneous disease. Informed consent was obtained from each patient before skin biopsy.

Single 4-mm cutaneous punch biopsies were obtained under local anesthesia. From the first set of 7 patients with respiratory allergy, a total of 22 two-mm skin biopsies were obtained at $20 \mathrm{~min}, 2,4$, or $24 \mathrm{~h}$ after intradermal injection with either saline or allergen (ragweed or dust mite). To rule out the possibility of nonspecific stimulation with allergen, we also studied a total of 8 skin biopsies from 4 healthy controls after local allergen injection. These specimens were immediately flash frozen for analysis by immunohistochemistry (see below). From a 
second set of 8 patients with respiratory allergy, 2-mm skin biopsies were obtained from either untreated skin sites $(n=22)$ or skin sites obtained 20 min after injection with either saline or allergen $(n=4)$. These specimens were subjected to organ culture before analysis by immunohistochemistry (see below).

Immunohistochemical studies. Immunohistochemical staining was carried out as previously described (9). All biopsies were flash frozen in optimal cooling temperature (OCT) compound (Miles Laboratories Inc., Naperville, IL) in liquid nitrogen and stored at $-70^{\circ} \mathrm{C}$, until cryosectioning. $6-\mu \mathrm{m}$ cryostat sections were cut, fixed in acetone for $10 \mathrm{~min}$, washed 3 times in phosphate-buffered saline, and incubated for $1 \mathrm{~h}$ at room temperature with the monoclonal antibody. Sections were then washed and incubated with peroxidase-conjugated rabbit anti-mouse IgG (Dako Corp., Santa Barbara, CA), diluted in phosphate-buffered saline containing $20 \%$ normal human serum for $60 \mathrm{~min}$. Peroxidase activity was detected using acetylethylcarbazol $(0.002 \%$ in $5 \%$ dimethylformamide, $0.2 \%$ sodium acetate buffer, $\mathrm{pH} 5$, with $0.03 \% \mathrm{H}_{2} \mathrm{O}_{2}$ ). Sections were then washed in distilled water, lightly poststained with hematoxylin, and mounted. The anti-ELAM-1 monoclonal antibody used in these studies was $\mathrm{H4} / 18$, which is $\operatorname{IgG}_{1} \kappa(10)$. Control antibodies were routinely tested on step sections of the same tissue specimens. The negative staining control was an irrelevant $\operatorname{IgG}_{1} \kappa$ and the positive control for EC staining was anti-von Willebrand factor $\left(\operatorname{IgG}_{1}\right.$, Dako Corp.). In all experiments, these antibodies were also used to stain a specimen of acute appendicitis for ELAM-1 positive venules.

Grading of stained sections was initially done without knowledge of the identity of the patient or the time of biopsy. Assessment of staining for ELAM-1, which is absent in normal endothelium, was performed by counting positively staining vessels, and the staining intensity was graded as either 0 (absent), $1+$ (weak), $2+$ (moderate), $3+$ (strong), or $4+$ (very strong).

Organ cultures. Skin biopsies from nonlesioned skin of patients with respiratory allergy were immediately immersed into RPMI 1640 culture medium (Flow Laboratories, McLean, VA) supplemented with $10 \%$ (vol/vol) fetal calf serum, $1 \%$ penicillin/streptomycin, and $10 \mathrm{mM}$ L-glutamine (Gibco Chemical Co., Grand Island, NY). Explants were incubated at $37^{\circ} \mathrm{C}$ with humidified $5 \% \mathrm{CO}_{2} / 95 \%$ air for $5 \mathrm{~h}$ before harvesting. In selected experiments, organ cultures were preincubated with either $200 \mathrm{U} / \mathrm{ml}$ of rabbit anti-human IL- $1 \alpha$ and $\beta$ (Endogen) and/or $500 \mathrm{U}$ of rabbit anti-human TNF- $\alpha$ (Genzyme, Boston, MA) for $1 \mathrm{~h}$ before the addition of allergen. Replicate explants were also incubated in identical concentrations of nonimmune rabbit serum to exclude nonspecific interference with local cytokine release. After harvesting the explants, all tissue specimens were flash frozen and processed for immunochemical staining as described above.

\section{Results}

Endothelial activation in situ in elicited late phase reactions. Our previous studies have demonstrated that, using antibody $\mathrm{H} 4 / 18$, normal skin does not show any reactivity for the ELAM-1 antigen (9). In these experiments, we studied 7 patients with respiratory allergy known to have LPR after intradermal injection with either ragweed or dust mite allergen. A total of 22 skin biopsies were obtained either at $20 \mathrm{~min}, 2,4,6$, or $24 \mathrm{~h}$ after intradermal injection with either saline or allergen. Saline injected sites in 4 of 4 atopic subjects tested did not express ELAM-1 antigen (Fig. 1 a). In contrast, in 7 of 7 atopic patients, allergen induced the appearance of ELAM-1 on EC (Fig. $1 b$ ). ELAM-1 expression occurred concurrently with the development of inflammatory cell infiltrates between $2-4 \mathrm{~h}$ after intradermal injection (Fig. 2). The intensity of ELAM-1 expression peaked at $6 \mathrm{~h}$ and was significantly decreased $24 \mathrm{~h}$ after intradermal allergen injection. Because histological studies were performed on frozen (rather than paraffin-embedded) sections in these small biopsies, the cell types in the inflamma-
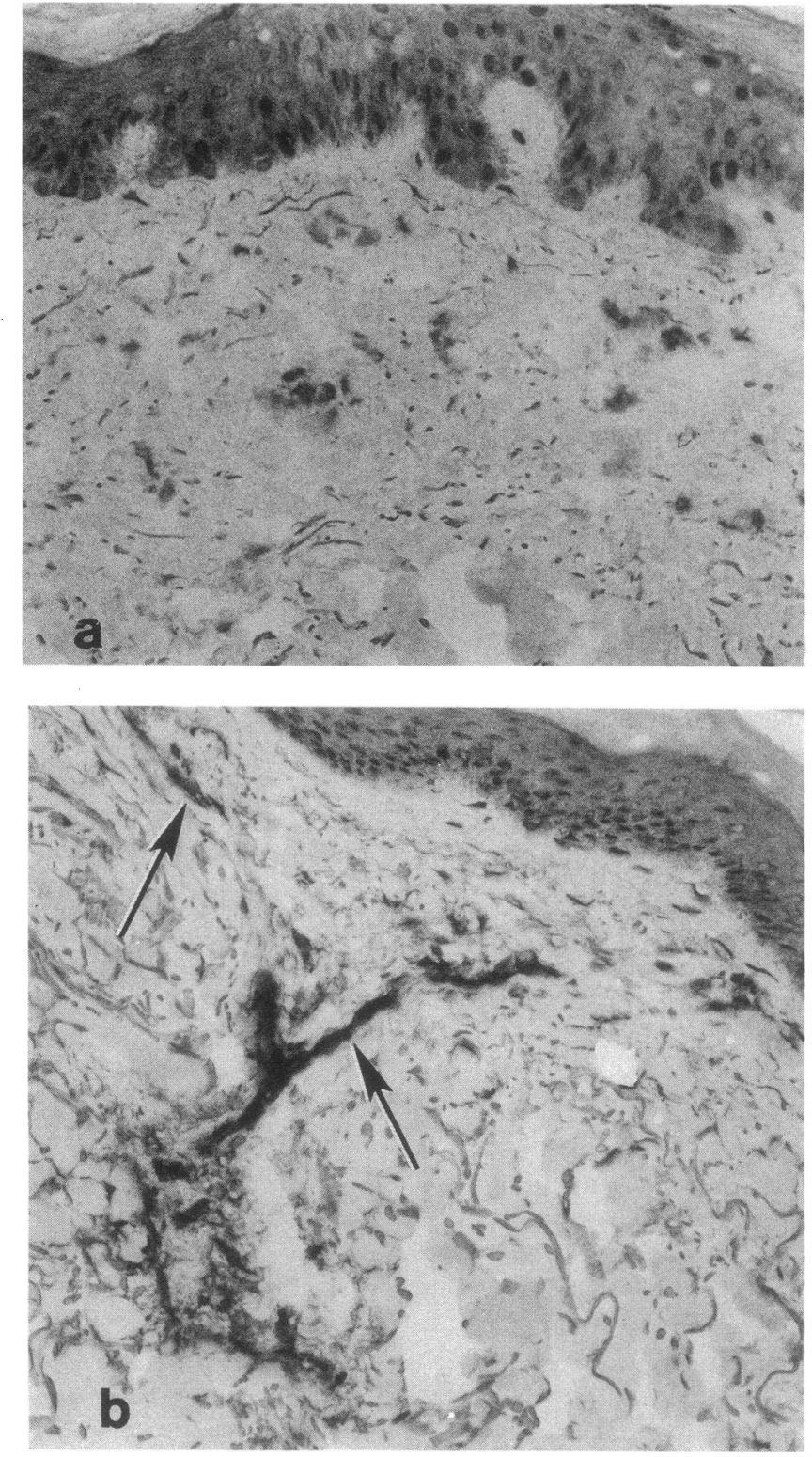

Figure 1. Skin biopsies from LPR stain for ELAM-1. (a) Skin biopsy obtained $4 \mathrm{~h}$ after intradermal saline injection in a patient with respiratory allergy stained for ELAM-1. Vessels in the dermis do not stain for ELAM-1. (b) Skin biopsy $4 \mathrm{~h}$ after in vivo induction of a late phase reaction by ragweed antigen in a patient with respiratory allergy stained for ELAM-1. Vessels in the dermis are positively stained for ELAM-1 and are associated with early inflammatory cell infiltrate.

tory infiltrate could not be precisely quantitated. I he earnest lesions at $2 \mathrm{~h}$ contained polymorphonuclear as well as mononuclear cells, but predominantly the former. At $6 \mathrm{~h}$, both cell types continued to be present, and in the 24-h lesions, the cellular infiltrate consisted largely of mononuclear cells. Occasionally eosinophils were present at all time intervals.

We also examined the intensity of ELAM-1 expression in skin biopsies obtained $6 \mathrm{~h}$ after intradermal injection of ragweed and dust mite allergen in separate skin sites of four nonatopic healthy controls. As shown in Fig. 2, the intensity of ELAM-1 expression in skin biopsies taken from allergen-in- 


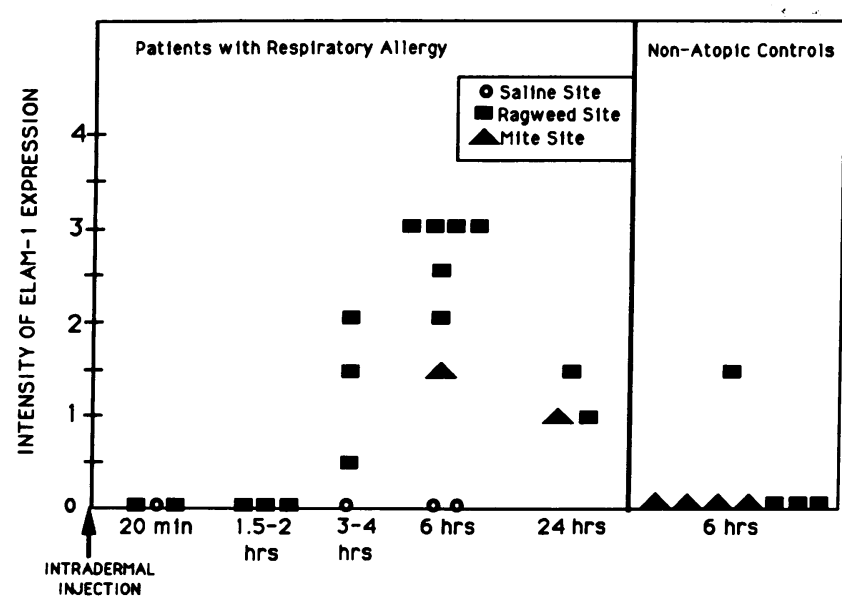

Figure 2. Time course of ELAM-1 expression during elicited LPR in patients with respiratory allergy. ELAM-1 expression was evident after 3-4 h, increased in intensity at $6 \mathrm{~h}$, and decreased at $24 \mathrm{~h}$. The two biopsies of mite-injected sites from atopic individuals were obtained from the same individual. Only 1 of 7 sites in 4 nonatopic controls showed ELAM-1 staining, and saline sites were uniformly negative.

jected skin sites of nonatopic controls was significantly less than that observed in atopic skin. Specifically, the $P$ value was $<0.01$ using a Wilcoxon Rank Sum test comparing the intensity of ELAM-1 expression at the 6-h timepoint in allergen-injected skin sites of atopic individuals $(n=7)$ versus nonatopic controls $(n=8)$. The single positive response to ragweed was in a patient who complained of respiratory allergy but was skin test negative by gross examination after prick and intradermal injection to ragweed. These results suggest that the injection of allergen into skin does not act directly on EC to cause expression of ELAM-1 but rather acts by inducing an immune response in atopic individuals.

Endothelial activation in skin organ cultures. In this series of experiments, we wished to determine whether the induction of ELAM-1 during LPR resulted primarily from signals derived from resident skin cells, or secondarily as a result of the inflammatory cell infiltrate. Two approaches were used to examine these possibilities. First, we obtained skin biopsies from two atopic individuals, 20 min after either an allergen injection or saline injection using the same protocol followed for in situ allergen responses. Based on the previous experiments, neither ELAM-1 expression nor leukocyte infiltrates are expected at this early time postinjection. These biopsies were placed into organ culture without further exposure to allergen. After $6 \mathrm{~h}$ of culture, these skin explants were frozen and stained for ELAM1 expression. EC in the biopsies obtained from the ragweed- or dust mite-injected skin sites of these two donors both developed marked (3+) ELAM-1 expression. In contrast, skin biopsies from both donors injected in vivo with saline had only minimal reactivity $(0.5+)$ for ELAM- 1 . These initial experiments suggested that resident skin cells rather than infiltrating cells are the source of cytokine induced EC activation after intradermal allergen injection.

A second experimental approach was used to extend these observations. In these latter experiments, untreated skin biopsies taken from six different patients with respiratory allergy, were placed in organ culture and exposed in vitro either to ragweed or dust mite allergen, or to saline for $5 \mathrm{~h}$. In each case, in vitro exposure to allergen but not saline resulted in significant ELAM-1 expression on EC (Fig. 3). Hematoxylin staining confirmed that the ELAM-1 expression observed on these tissue sections occurred without an inflammatory infiltrate.

Expression of ELAM-1 is IL-1 and TNF dependent. ELAM-1 expression can be induced in skin organ culture by exogenous IL-1 or TNF (11). To test whether these cytokines mediate the response to allergen, skin explants were preincubated for $1 \mathrm{~h}$ with polyclonal blocking antiserum to human TNF- $\alpha(500 \mathrm{U} / \mathrm{ml})$ and/or anti-human IL- $\alpha$ and $\beta(200 \mathrm{U} /$ $\mathrm{ml}$ ), followed by $5 \mathrm{~h}$ more of organ culture after addition of allergen. As shown in Table I, preincubation with anti-human TNF- $\alpha$ alone, anti-IL- $1 \alpha$ and $\beta$ alone, or nonimmune rabbit serum failed to prevent in vitro ELAM-1 induction by allergen (Table I and Fig. $4 a$ ). However in four separate explants (patients 3, 4, 5, and 6 in Table I), preincubation of skin explants with a combination of anti-IL-1 and anti-TNF completely abrogated expression of in vitro allergen-induced ELAM- 1 expression (Fig. $4 \mathrm{~b}$ ). These results suggest that the release of endogenous IL- 1 and TNF- $\alpha$ associated with allergen exposure appear to be responsible for elicitation of endothelial activation, as defined by ELAM-1 induction.

\section{Discussion}

The LPR plays an important pathogenic role in allergic diseases. LPR have been demonstrated in patients who have

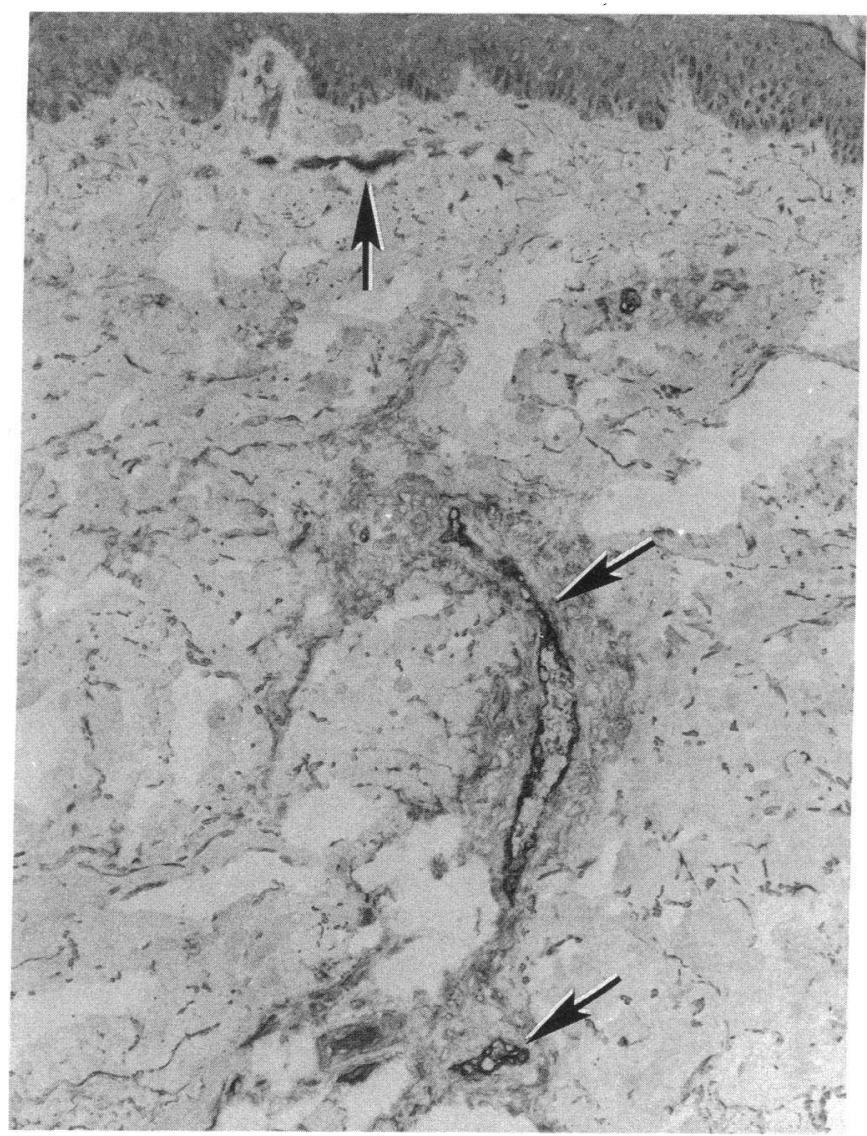

Figure 3. Skin vascular endothelial cells express ELAM-1 after allergen exposure in vitro. Skin explant from an atopic patient cultured in the presence of allergen for $5 \mathrm{~h}$ and stained for ELAM-1, showing ELAM-1 staining in the endothelium. 
Table I. Cytokine Dependence of Allergen-induced ELAM-1 Expression

\begin{tabular}{clll}
\hline Experiment:Part & Allergen & \multicolumn{1}{c}{ Antibody } & $\begin{array}{c}\text { ELAM-1 } \\
\text { Expression }\end{array}$ \\
\hline \multirow{2}{*}{$1: 1$} & None & None & $1+$ \\
& Ragweed & None & $2.5+$ \\
& Ragweed & Anti-TNF- $\alpha$ & $2+$ \\
& Ragweed & Anti-TNF- $\beta$ & $3+$ \\
$1: 2$ & None & None & $0.5+$ \\
& Dust mite & None & $2+$ \\
& Dust mite & Anti-TNF- $\alpha$ & $1.5+$ \\
& Dust mite & Anti-TNF- $\beta$ & $2+$ \\
$2: 3$ & None & None & 0 \\
& Ragweed & None & $2+$ \\
& Ragweed & Anti-TNF- $\alpha+$ anti-IL-1 & 0 \\
$2: 4$ & None & None & 0 \\
& Ragweed & None & $2+$ \\
& Ragweed & Anti-TNF- $\alpha+$ anti-IL-1 & 0 \\
$3: 5$ & Ragweed & Nonimmune rabbit serum & 1.5 \\
& Ragweed & Anti-TNF- $\alpha$ & $2+$ \\
& Ragweed & Anti-IL-1 & 1.5 \\
& Ragweed & Anti-TNF- $\alpha+$ anti-IL-1 & 0 \\
& Ragweed & Nonimmune RS & $2+$ \\
& Ragweed & Anti-TNF- $\alpha$ & $2+$ \\
& Ragweed & Anti-IL-1 & $2+$ \\
& Ragweed & Anti-TNF- $\alpha+$ anti-IL-1 & 0 \\
& & & \\
& & &
\end{tabular}

Skin explants from patients with respiratory allergy were stimulated in vitro for $5 \mathrm{~h}$ with allergen in the presence of either nonimmune rabbit serum, anti-TNF- $\alpha$, anti-IL-1, or a mixture of anti-TNF and anti-IL- 1 as described in Methods.

asthma and allergic rhinitis $(3,4,12)$. The intensity of nonspecific bronchial hyperreactivity in asthmatic reactions after antigen bronchoprovocation has been found to be proportional to the intensity of the LPR (13). Clinical improvement in asth-
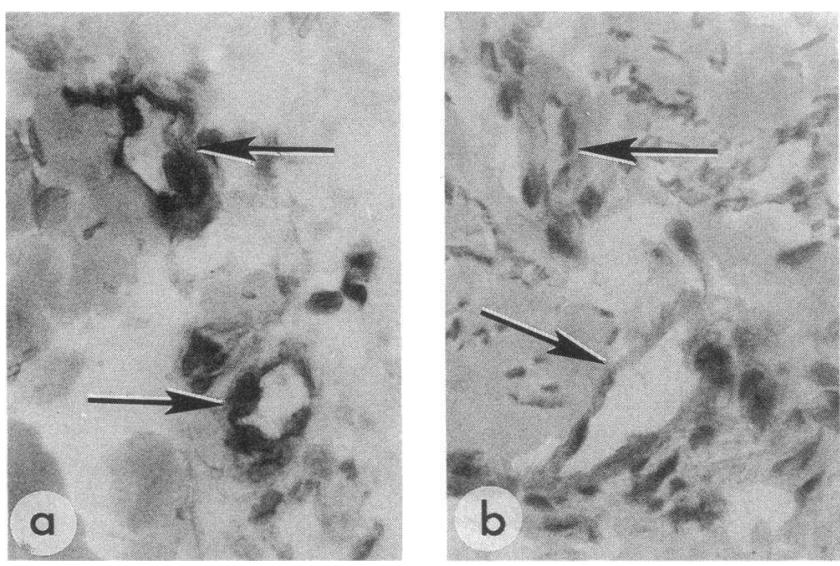

Figure 4. Expression of ELAM-1 in vitro is blocked by anti-IL-1 plus anti-TNF. (a) High power of two blood vessels from a skin explant cultured with allergen for $5 \mathrm{~h}$ in the presence of normal rabbit serum. (b) A similar explant cultured in the presence of anti-IL-1 plus antiTNF- $\alpha$ in the medium. Note the absence of endothelial staining. matic symptoms after allergen immunotherapy correlates with a marked attenuation of LPR after bronchoprovocation challenge (14). The immunopathologic events that result in the inflammatory infiltrate associated with the LPR are poorly understood. The accessibility of the skin for in situ investigation makes it an important model for understanding the pathogenesis of allergic LPR in other organs.

The induction of endothelial leukocyte adhesion molecules represent an important initial event in the accumulation of inflammatory cells at the site of antigen stimulated tissues (10, 15). In our study of allergen-induced LPR, we found that the ELAM-1 antigen occurs concurrently with the development of inflammatory cell infiltrates 2-4 h after elicitation of the LPR in vivo. Furthermore, the local secretion of IL-1 and TNF- $\alpha$ are required for the induction of ELAM-1 expression.

In the skin, IL- 1 and TNF- $\alpha$ may be produced by several cell types. Keratinocytes produce IL-1 and monocytes produce both IL-1 and TNF- $\alpha$ (16). Vascular smooth muscle cells also produce small amounts of IL-1 and $\operatorname{TNF}(17,18)$ and EC themselves synthesize IL-1, although little is secreted (19). Interleukin 3-dependent and -transformed mast cell lines have recently been demonstrated to release multiple cytokines after cross-linking of their high affinity IgE (FceRI) receptors via the interaction of $\operatorname{IgE}$ with specific multivalent allergens $(20,21)$. These mast cell-derived cytokines include IL-1, TNF- $\alpha$, IL-3, IL-4, IL-5, IL-6, and GM-CSF. Mast cells are located around the superficial dermal venules, the sites of most cutaneous inflammatory responses, and the vascular segments that most prominently express ELAM-1 during an elicited LPR. The immediate release of cytokines after allergen-induced mast cell degranulation is most compatible with the data presented in this study. This interpretation is supported by the recent studies of Klein et al. (22) who reported that dermal EC expressed ELAM-1 $2 \mathrm{~h}$ after in vitro degranulation of human mast cells using anti-IgE in skin organ cultures. These investigators were able to completely block anti-IgE-induced expression of ELAM-1 using antiserum to TNF- $\alpha$. In contrast, we were unable to significantly inhibit allergen-induced ELAM-1 expression with anti-TNF- $\alpha$ alone but required the combination of both TNF- $\alpha$ and anti IL-1. A role for IL-1 as well as TNF is consistent with the recent report by Charlesworth et al. (23), using a blister chamber model, that allergen induced LPR is accompanied by early production of IL-1.

We conclude that cytokine-induced EC activation plays a role in the migration of inflammatory cells into the allergen-induced LPR. Furthermore, cytokines derived from resident cells in the skin, probably mast cells rather than infiltrating leukocytes, appear to mediate the EC activation. The cytokines in question are likely to be IL-1 and/or TNF- $\alpha$. Therapeutic strategies that seek to reduce the inflammatory cascade associated with allergic diseases will need to consider the role of local cytokine secretion during the LPR.

\section{Acknowledgments}

We wish to thank Maureen Sandoval for her assistance in the preparation of this manuscript.

This work was supported in part by grants from the National Institutes of Health (HL-37260 and HL-36028). Dr. Pober was an Established Investigator of the American Heart Association when these studies were conducted. 


\section{References}

1. Dolovich, J., F. E. Hargreave, R. Chalmers, K. J. Shier, J. Gauldie, and J. Bienenstock. 1973. Late cutaneous allergic responses in isolated IgE-dependent reactions. J. Allergy Clin. Immunol. 52:38-46.

2. Gleich, G. J. 1982. The late phase of the immunoglobulin E-mediated reaction: a link between anaphylaxis and common allergic disease? J. Allergy Clin. Immunol. 70:160-169.

3. Cockcroft, D. W., and K. Y. Murdock. 1987. Comparative effects of inhaled salbutamol, sodium cromoglycate, and beclomethasone dipropionate on allergen-induced early asthmatic responses, late asthmatic responses, and increased bronchial responsiveness to histamine. J. Allergy Clin. Immunol. 79:734740.

4. Durham, S. R., T. H. Lee, O. Cromwell, R. J. Shaw, T. G. Merrett, J. Merrett, P. Cooper, and A. B. Kay. 1984. Immunologic studies in allergen-induced late-phase asthmatic reactions. J. Allergy Clin. Immunol. 74:49-60.

5. Pober, J. S., and R. S. Cotran. 1990. Cytokines and endothelial cell biology. Physiol. Rev. 70:427-451.

6. Pober, J. S., and R. S. Cotran. 1990. The role of endothelial cells in inflammation. Transplantation. 50:537-544.

7. Bevilacqua, M. P., J. S. Pober, D. L. Mendrick, R. S. Cotran, and M. A. Gimbrone, Jr. 1987. Identification of an inducible endothelial-leukocyte adhesion molecule. Proc. Natl. Acad. Sci. USA. 84:9238-9242.

8. Pober, J. S., M. A. Gimbrone Jr., L. A. Lapierre, D. L. Mendrick, W. Fiers, R. Rothlein, and T. A Springer. 1986. Overlapping patterns of activation of human endothelial cells by interleukin 1, tumor necrosis factor and immune interferon. J. Immunol. 137:1893-96.

9. Cotran, R. S., M. A. Gimbrone Jr., M. P. Bevilacqua, D. L. Mendrick, and J. S. Pober. 1986. Induction and detection of a human endothelial activation antigen in vivo. J. Exp. Med. 164:661-666.

10. Pober, J. S., M. P. Bevilacqua, D. L. Mendrick, L. A. LaPierre, W. Fiers, and M. A. Gimbrone, Jr. 1986. Two distinct monokines, interleukin 1 and tumor necrosis factor each independently induce biosynthesis and cell surface expression of the same antigen on the surface of cultured human vascular endothelial cells. J. Immunol. 136:1680-1687.

11. Messadi, D. V., J. S. Pober, W. Fiers, M. A. Gimbrone, Jr., and G. F. Murphy. 1987. Induction of an activation antigen on post-capillary venular endothelium in human skin organ culture. J. Immunol. 139:1557-1562.
12. Bascom, R., M. Wachs, R. M. Naclerio, U. Pipkorn, S. J. Galli, and L. M. Lichtenstein. 1988. Basophil influx occurs after nasal challenge: effects of topical corticosteroid pretreatment. J. Allergy Clin. Immunol. 81:580-589.

13. Cartier, A., N. C. Thomson, P. A. Frith, R. Roberts, and F. E. Hargreave. 1982. Allergen-induced increase in bronchial responsiveness to histamine: relationship to the late asthmatic response and change in airway caliber. J. Allergy Clin. Immunol. 70:170-177.

14. Warner, J. O., J. F. Soothill, J. F. Price, and E. N. Hey. 1978. Controlled trial of hyposensitization with Dermatophagoides pteronyssinus antigen in children with asthma. Lancet. ii:912-917.

15. Cotran, R. S., and J. S. Pober. 1988. Endothelial activation: its role in inflammatory and immune reactions. In Endothelial Cell Biology. N. Simionesc and M. Simionescu, editors. Plenum Publishing Corp., New York. 335-347.

16. Kupper, T. S. 1989. Production of cytokines by epithelial tissues. A new model for cutaneous inflammation. Am. J. Dermatopathol. 11:69-73.

17. Libby, P., J. M. Ordovas, L. K. Birinyi, K. R. Auger, and C. A. Dinavello. 1986. Inducible interleukin-1 gene expression in human vascular smooth muscle cells. J. Clin. Invest. 78:1432-1438.

18. Warner, S. J. C., and P. Libby. 1989. Human vascular smooth muscle cells: target for and course of tumor necrosis factor. J. Immunol. 142:100-109.

19. Kurt-Jones, E. A., W. Fiers, and J. S. Pober. 1987. Membrane interleukin 1 induction on human endothelial cells and dermal fibroblasts. J. Immunol. 139:2317-2324.

20. Burd, P. R., H. W. Roger, J. R. Gordon, C. A. Martin, S. Jayaraman, S. D. Wilson, A. M. Dvorak, S. J. Galli, and M. E. Dorf. 1989. Interleukin 3-dependent and -independent mast cells stimulated with IgE and antigen express multiple cytokines. J. Exp. Med. 170:245-257.

21. Plaut, M., J. H. Pierce, C. J. Watson, J. Hanley-Hyde, R. P. Nordan, and W. E. Paul. 1989. Mast cell lines produce lymphokines in response to cross-linkage of FceRI or to calcium ionophores. Nature (Lond.). 339:64-67.

22. Klein, L. M., R. M. Lavker, W. L. Matis, and G. F. Murphy. 1989 Degranulation of human mast cells induces an endothelial antigen central to leukocyte adhesion. Proc. Natl. Acad. Sci. USA. 86:8972-8976.

23. Charlesworth, E. N., B. S. Bochner, L. M. Lichtenstein, S. Gillis, C. Dinarello, and R. P. Schleimer. 1990. Interleukin-1 release during the cutaneous latephase response to antigen. J. Allergy Clin. Immunol. 85:266a. (Abstr.) 